\title{
TAIGA-HiSCORE detection of the CATS-LIDAR on the ISS as fast moving point source
}
A.Porelli ${ }^{8}$, R.Wischnewski ${ }^{8 *}$, A.Garmash ${ }^{11,12}$, I. Astapov ${ }^{9}$, P. Bezyazeekov ${ }^{2}$, V. Boreyko ${ }^{10}$, A. Borodin ${ }^{10}$, M. Brueckner ${ }^{8}$, N. Budnev ${ }^{2}$, A. Chiavassa ${ }^{4}$, A. Dyachok ${ }^{2}$, O. Fedorov ${ }^{2}$, A. Gafarov ${ }^{2}$, E. Gorbovskoy ${ }^{16}$ N. Gorbunov ${ }^{10,14}$, V. Grebenyuk ${ }^{10,14}$, O. Gress ${ }^{2}$, T. Gress ${ }^{2}$, O. Grishin ${ }^{2}$, A. Grinyuk ${ }^{10}$, D. Horns ${ }^{6}$, A. Ivanova ${ }^{2}$, N. Kalmykov ${ }^{1}$, Y. Kazarina² ${ }^{2}$, V. Kindin ${ }^{9}$, P. Kirilenko ${ }^{11,12}$, S. Kiryuhin ${ }^{2}$, R. Kokoulin ${ }^{9}$, K. Kompaniets ${ }^{9}$, E. Korosteleva ${ }^{1}$, V. Kozhin ${ }^{1}$, E. Kravchenko ${ }^{11,12}$, M. Kunnas ${ }^{6}$, L. Kuzmichev ${ }^{1,2}$, Yu. Lemeshev ${ }^{2}$, V. Lenok ${ }^{2}$, V. Lipunov ${ }^{16}$, B. Lubsandorzhiev ${ }^{1,3}$, N. Lubsandorzhiev ${ }^{1}$, R. Mirgazov ${ }^{2}$, R. Mirzoyan ${ }^{5,2}$, R. Monkhoev ${ }^{2}$, R. Nachtigall ${ }^{6}$, E. Osipova ${ }^{2}$, A. Pakhorukov ${ }^{2}$, M. Panasyuk ${ }^{1}$, L. Pankov², A. Petrukhin ${ }^{9}$, V. Poleschuk ${ }^{2}$, E. Popescu ${ }^{13}$, E. Popova ${ }^{1}$, E. Postnikov ${ }^{1}$, V. Prosin ${ }^{1}$, V. Ptuskin 7 , E. Rjabov ${ }^{2}$, G. Rubtsov ${ }^{3}$, A. Pushnin ${ }^{2}$, Y. Sagan ${ }^{10}$, V. Samoliga ${ }^{2}$, Yu. Semeney ${ }^{2}$, A. Silaev ${ }^{1}$, A. Silaev(junior) ${ }^{1}$, A. Sidorenkov ${ }^{3}$, A. Skurikhin ${ }^{1}$, V. Slunecka ${ }^{10}$, A. Sokolov ${ }^{11,12}$, G. Spengler ${ }^{15}$, C. Spiering ${ }^{8}$, L. Sveshnikova ${ }^{1}$, V. Tabolenko ${ }^{2}$, B. Tarashansky ${ }^{2}$, A. Tkachenko ${ }^{10}$, L. Tkachev ${ }^{10,14}$, M. Tluczykont ${ }^{6}$, A. Zagorodnikov ${ }^{2}$, D. Zhurov ${ }^{2}$, V. Zurbanov ${ }^{2}$, I. Yashin ${ }^{9}$
${ }^{1}$ Skobeltsyn Institute of Nuclear Physics MSU, Moscow, Russia, ${ }^{2}$ Institute of Applied Physics ISU, Irkutsk, Russia, ${ }^{3}$ Institute for Nuclear Research of RAN, Moscow, Russia, ${ }^{4}$ Dipartimento di Fisica Generale Universiteta di Torino and INFN, Torino, Italy, ${ }^{5}$ Max-Planck-Institute for Physics, Munich, Germany, ${ }^{6}$ Institut für Experimentalphysik, University of Hamburg, Germany, ${ }^{7}$ IZMIRAN, Moscow, Russia, ${ }^{8}$ Deutsches Elektronen Synchrotron (DESY), Zeuthen, Germany, ${ }^{9}$ National Research Nuclear University MEPhI, Moscow, Russia, ${ }^{10}$ JINR, Dubna, Russia, ${ }^{11}$ Novosibirsk State University, NSU, Novosibirsk, Russia, ${ }^{12}$ Budker Institute of Nuclear Physics SB RAS, Novosibirsk, Russia, ${ }^{13}$ ISS, Bucharest, Romania, ${ }^{14}$ Dubna State University, Dubna, Russia, ${ }^{14}$ Dubna State University, Dubna, Russia, ${ }^{15}$ Institute for Physics, Humboldt University, Berlin, Germany ${ }^{16}$ Sternberg Astronomical Institute, Moscow State University, Moscow, Russia; E-mail: ralf.wischnewskiedesy.de

We report the first ground-based observation of the CATS-LIDAR onboard the ISS by the TAIGAHiSCORE gamma-ray facility, and the MASTER-Tunka Robotic telescope. HiSCORE detects unscattered laser light directly from the ISS, at up to km-scale distance from the laser beam spot on ground. The ISS-LIDAR turns out to be a unique calibration tool, in particular to verify the absolute astronomical pointing of HiSCORE.

We detected the LIDAR for 11 ISS-passages; among these were observations of forward scattering of the laser beam in dense clouds, which might carry information complementary to the LIDAR technique. We expect other air Cherenkov installations like IACTs to benefit from this light source.

35th International Cosmic Ray Conference - ICRC2017

10-20 July, 2017

Bexco, Busan, Korea 


\section{Introduction}

TAIGA-HiSCORE is a Cherenkov shower-front timing array, under construction at the Tunka site in Siberia, and part of the hybrid TAIGA facility for cosmic-ray and gamma-ray physics. TAIGA-HiSCORE is today a $0.25 \mathrm{~km}^{2}$ detector array comprising 28 optical detector stations with $\sim 0.5 \mathrm{~m}^{2}$ sensitive area and $0.6 \mathrm{sr}$ acceptance each, to detect ns-duration Cherenkov light flashes from gamma ray showers of $\mathrm{E}>50 \mathrm{TeV}$, arriving at the array within $<2 \mu$ s. For details see [1]-[5].

Precision pointing for shower reconstruction is a key to detect astrophysical gamma-ray sources in HiSCORE [7]. This paper describes a unique method to verify, in the absence of strong detected astrophysical sources, the absolute HiSCORE pointing using as artificial light source the $532 \mathrm{~nm}$ laser of the CATS-LIDAR on the International Space Station (ISS), which was serendipitously detected in the 2015/16 HiSCORE data set [6]. Absolute pointing calibration is based on the simultaneous observation by the robotic optical telescope MASTER-Tunka [12]. In addditon, ISS-events reflect the status of the atmoshphere, for some occasions scattering in clouds at $\mathrm{km}$-scale distance has been detected.

ISS-CATS detection is now part of the standard observation and calibration program of HiSCORE.

\section{HiSCORE observation of the CATS-LIDAR onboard the ISS}

Routine analysis of the 2015/16 data set revealed a few $\sim 1$ second intervals of very high array trigger rates, with reconstructed shower directions close to zenith, resembling a fast moving point source of angular speed $\sim 1 \mathrm{deg} / \mathrm{s}$. The event directions coincide with the trajectory of the ISS, passing almost vertically over the HiSCORE location, see the zoomed skyplot in Fig.1(a). As the strong light source we identified the CATS-LIDAR (Cloud Aerosol Transport System) [11] onboard the ISS at $400 \mathrm{~km}$ height, emitting $\sim 1 \mathrm{~mJ}$ per pulse at $532 \mathrm{~nm}$ with $4 \mathrm{kHz}$ repetition rate and $10 \mathrm{~ns}$ pulse duration. The beam points almost vertically downwards at small inclination (typically $1-2^{\circ}$ backwards), and diverges to a $14 \mathrm{~m}$ diameter spot on the ground.

The time-characteristics of the light signals, detected by the array during ISS passages ("ISSevents") are strikingly different from air shower events (EAS), which have a curved shower front: these are perfectly described by a plane light wave hitting HiSCORE, as described below. The observations are compatible with detection of the direct, unscattered LIDAR light component at distances of up to few $\mathrm{km}$ from the 1/e beam spot diameter on ground (footprint or "FFOV" [11]). We note, that for the given $\sim 1$ ns time resolution of HiSCORE, a spherical light front is indistinguishable from a plane wave for source distances beyond $80 \mathrm{~km}$; thus the result is compatible with the CATS-LIDAR being the point-like light source.

For a total of 11 ISS-passages in the seasons 2015/16 and 2016/17, LIDAR-light was detected in HiSCORE. Table 1 gives the characteristics for all 2016/17 passages, with six detections in eight favorable ISS-passages. The example in Fig. 1 shows the color-coded time-evolution for one passage in a 5-degree skyplot, together with the calculated ISS track. An extreme counting rate increase and periodic laser pulses are shown in the inserts of Fig.2.

\footnotetext{
* Speaker.
} 
The above described ISS-passages with detection of direct, unscattered light, are complemented with more complicated situations, with the passage or parts of it affected by scattering. An interesting situation is shown in Fig.3 for 2015/12/06, where the usual direct light is detected in the central 5-degree skymap (left). At larger zenith angles $\left(10^{\circ}-30^{\circ}\right)$ for about $200 \mathrm{~ms}$ a fast moving light beam is detected - which is the ISS-laser beam hitting clouds at $10 \mathrm{~km}$ height and km-scale distance from HiSCORE, generating a pointlike source moving at ISS-speed $(7.7 \mathrm{~km} / \mathrm{s})$, and seen with large angular speed from HiSCORE. An approximate reconstruction of the emission height is shown in Fig.4 (prelim.), and is overlayed with the total backscatter data from CATS [11].

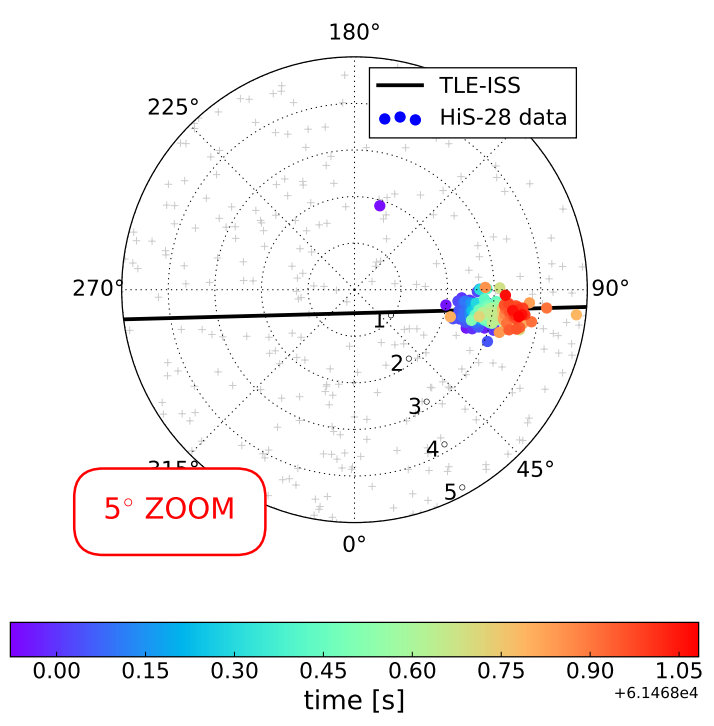

(a)

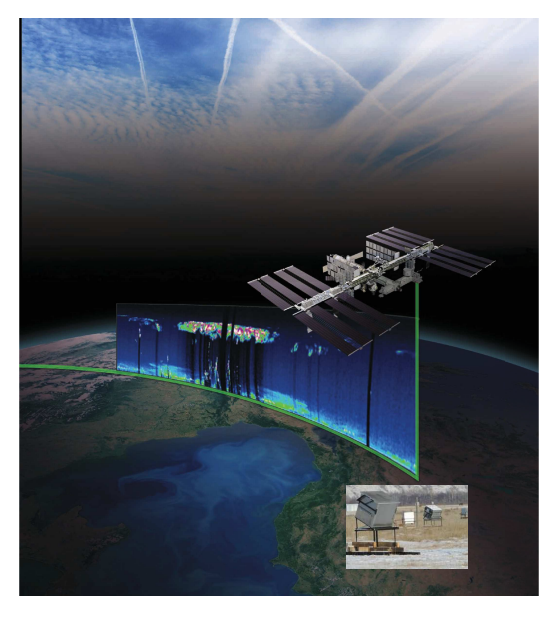

(b)

Figure 1: (a) Example of an observed ISS-passage (2017/03/25), color code gives time evolution within $\sim 1 \mathrm{sec}$. Grey crosses illustrate the reconstructed EAS directions, as recorded +-5 min around the ISS passage. (b) The Cloud Aerosol Transport System (CATS) operating from the ISS, with a LIDAR scanning the atmosphere profile (adapted from [11]).

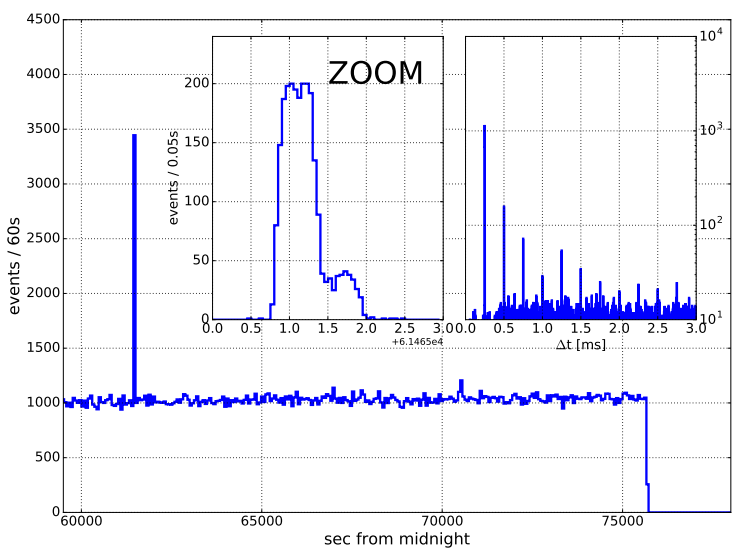

Figure 2: HiSCORE trigger rate for 2017/03/25. The insert shows a zoom of the 1 -sec ISS passage (with a DAQ-deadtime induced sharp drop at $1.5 \mathrm{~s}$ ); the $4 \mathrm{kHz}$ laser repetition gives an event time difference $\Delta \mathrm{t}$ with period of multiple of $250 \mu \mathrm{s}$. 


\begin{tabular}{c|c|c|c}
\hline \hline \multicolumn{4}{c}{ ISS passages for the 2016/17 Season } \\
\hline \hline UTC (pred) & $\delta$ (pred) $\left[{ }^{\circ}\right.$ ] & CATS Status & Observation $(+/-)$ \\
\hline 2016/09/27 16:29:04 & $89: 42$ & ON & + \\
2016/11/26 16:29:13 & $89: 39$ & ON & + \\
2017/01/25 16:30:21 & $89: 11$ & ON & + \\
2017/01/28 15:30:02 & $89: 46$ & ON & + \\
2017/03/22 18:05:00 & $88: 23$ & ON & - \\
2017/03/25 17:04:26 & $89: 29$ & ON & + \\
2017/03/28 16:03:50 & $89: 42$ & ON & - \\
2017/03/31 15:03:08 & $89: 03$ & ON & + \\
\hline
\end{tabular}

Table 1: Observation of the CATS-LIDAR for all ISS passages in the 2016/17 season, for $\delta>88^{\circ}$ and both HiSCORE and CATS operating; with time (UTC) and altitude $\delta$ as predicted for the closest zenith distance during the passage. LIDAR detection labeled as "+".

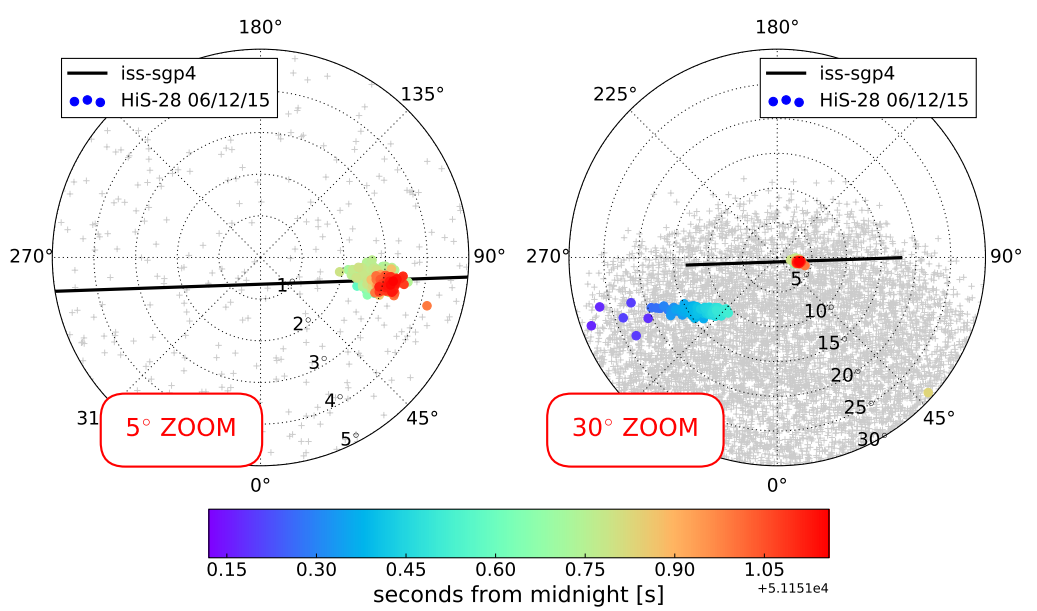

Figure 3: Different components of CATS-LIDAR light detected on 2015/12/06: (a) ISS events from direct light; (b) cloudscattered light events. (Grey crosses: +-5 min CR-events, see fig.1).

\section{Data anylsis}

\subsection{HiSCORE data analysis}

The "ISS events" are extracted from HiSCORE standard data processing, that merges stations triggered within $2 \mu \mathrm{s}$, and calibrates their time and amplitude signals. Arrival directions are reconstructed fitting the light front arrival times with a plane wave model. A reconstruction with a spherical front profile gives similar results. In this work, we use reconstructed angles $(\phi, \theta)$, UTC time and largest amplitude (MaxAmplitude), as found for each event.

\subsection{HiSCORE-MASTER combined analysis}

The robotic Telescope MASTER-Tunka [12], located $~ 500 \mathrm{~m}$ from HiSCORE, successfully recorded images of the full ISS/CATS-LIDAR passage on 2017/03/25 with its VWF camera, as shown in Fig.5(a)). We used this coincident, independent detection to check the HiSCORE absolute direction reconstruction (absolute pointing), by comparing both observations. Due to the close distance of the ISS and the different location of the two detectors, the coordinates (R.A., Dec) of the MASTER detection are first corrected by the parallax effect, and then transformed into 


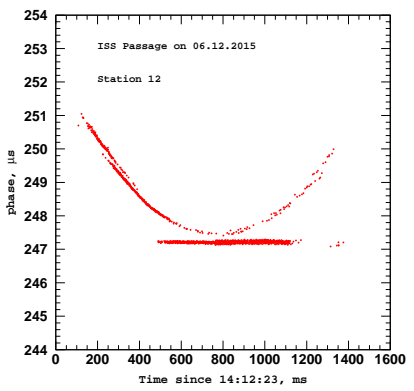

(a)

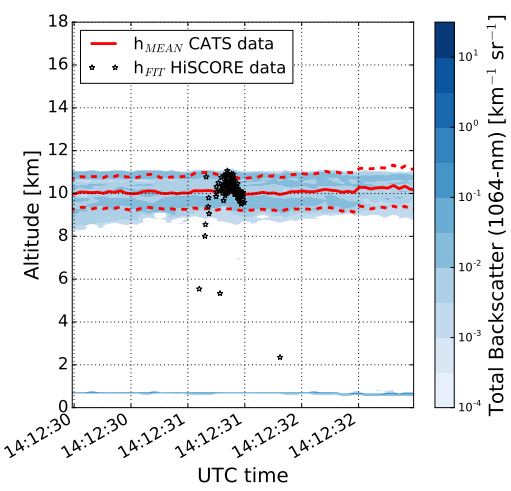

(b)

Figure 4: (a) Direct-light + cloud-scattered events, as seen by a phase-analysis for a single station. Plotted is the change in laser pulse-phase ( $f \sim 4 \mathrm{kHz}$ ), wrt to the central passage. Flat part: unscattered events; rising: cloud scattering gives additional ligth path delay. (b) Estimated cloud altitude (preliminary) using the reconstructed direction and the CATS-FFOV ground spot distance from HiSCORE.

the HiSCORE local (horizontal) system of reference (Fig.5(b)). We select the brightest parts of both observations: for HiSCORE events with MaxAmplitude $\geq 10^{5}$ (a.u.), and for MASTER the average coordinates of brightest pixels. We estimate the HiSCORE reconstruction mispointing, $\alpha_{M I S}$, by calculating the angular distance between the average reconstructed direction of the two detections. From this we obtain an upper limit on the HiSCORE mispointing of $\alpha_{\text {mis }} \sim 0.1^{\circ}$. Figure 5(b) shows the good alignment of the optical and HiSCORE images. We note, that for the first reported ISS/CATS detection [2], a preliminary time-calibration of the HiSCORE array was used, which resulted in a mispointing of $0.4^{\circ}$ and a small reconstruction offset from the ISS track. With improved time-calibration, showers coincide with the ISS path, see Figs.1(a) and 3.

From further coincident HiSCORE-MASTER observations (including also the high-resolution MASTER-II camera), we expect to improve the precision of this method, to finally allow a fine adjustment of the HiSCORE pointing.

\subsection{HiSCORE-CATS combined analysis}

For each detected passage, we perform a combined analysis between HiSCORE and CATS data [11], which contain position/orientation information on the ISS and the LIDAR beam as function of time. As a reference, the ISS orbital position in time is obtained from the NORAD TLEs data set (closest epoch to passage) [10], and the SGP4 model [9] is used to transform to the observer location. Between CATS data and SGP4 prediction we observe a systematic shift in time of $\delta t_{C A T S} \sim 1-2 \mathrm{sec}$, as shown by the curves in fig.6(a), giving the time-evolution of zenith for ISS and detected events. While the reason for this CATS-shift is still under investigation, we perform an ad-hoc correction by $\delta t_{C A T S}$. In turn, HiSCORE events (blue dots on Fig.6) are consistent with the ISS-TLE track, after an additional time-adjustment $\delta t_{T L E}$ of $0-0.7 \mathrm{~s}$ (average $0.2 \mathrm{~s}$ ), that aligns the peak of the HiSCORE amplitude development in fig.6(b) with the closest distance to the CATS spot on ground (CATS-FFOV). It seems, that a limited precision of ISS-TLE location/time is the origin for this additional $\delta t_{T L E}$, since HiSCORE UTC-times are well understood (GPSDO/RbClock controlled $[3,5])$. We regard results obtained for detailed geometry alignment etc as somewhat 
MASTER detection of ISS/CATS-LIDAR

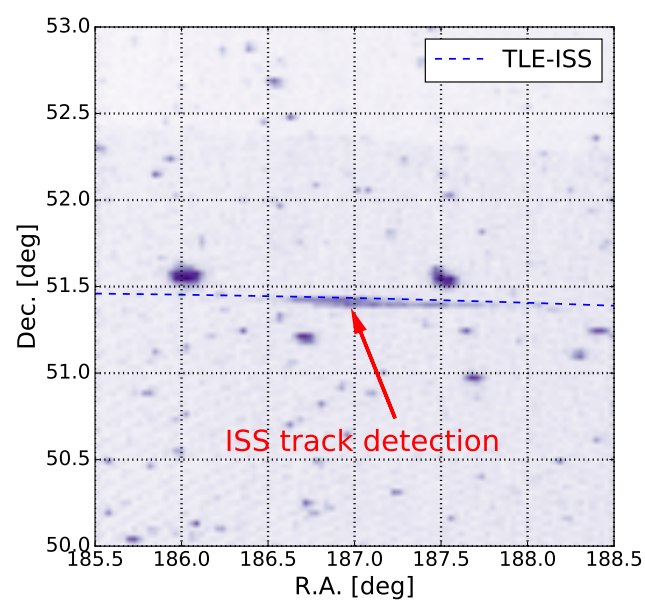

(a)

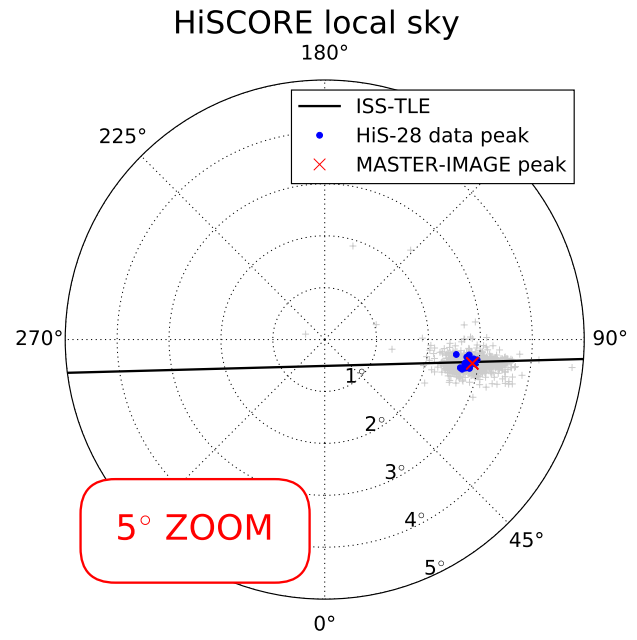

(b)

Figure 5: The simultaneous MASTER-HiSCORE detection of the ISS on 2017/03/25 (golden event). (a) Optical MASTER telescope detection of the CATS-LIDAR signal while the ISS passes through the field of view. The ISS-track is overlayed as dashed line on the processed image. The arrow points to the maximum detected intensity along the ISS track. (b) Local sky events as detected with HiSCORE (for brightest events). The red cross corresponds to the point of maximal intensity found on the MASTER image. The spatial coincidence of the point of maximal intensity in (a) and the HiSCORE events in (b) demonstrate the good pointing calibration of HiSCORE.

preliminary, until these effects are understood. Once precision location and timing for the ISS is established, all ISS passages can be used for precision calibration, like done for now for the HiSCORE-MASTER coincident observation in Fig.5.

\section{Application of the ISS-LIDAR}

The main application of the CATS-LIDAR onboard the ISS for HiSCORE is the absolute pointing calibration, as discussed in Sect.3.2, and is so far based on precision observation of the CATS/LIDAR by MASTER. Once high precision ISS track prediction will be available, pointing calibration is possible for all passages (including historical).

Furthermore, 'ISS events' in HiSCORE can be used like for a perfectly known "test beam" (using the strikingly simple and deterministic arrival time pattern, as compared to the complex and stochastic EAS amplitude-time patterns) to:

- determine the HiSCORE angular resolution, by comparing directions for neighbouring ISS pulses: emitted from almost identical locations (from ISS motion expect $1^{\circ} / \mathrm{s} / 4 \mathrm{kHz}<.001^{\circ}$ ). See Fig.8(a) for a preliminary analysis, giving about $0.1^{\circ}$ resolution for plane wave events,

- calibrate the HiSCORE-station relative time-offsets using ISS-events ("time-offset calibration", as discussed in [2, 8]). See Fig.8(b) for a comparison with other calibration methods,

- measure the time jitter for each station, see [2], 


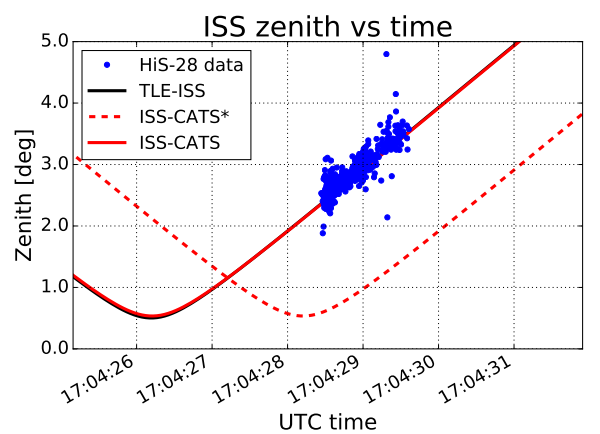

(a)

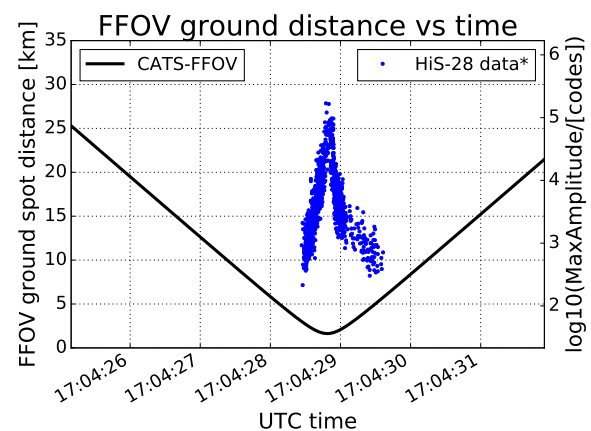

(b)

Figure 6: HiSCORE-CATS combined analysis. (a) Zenith angle vs. time for ISS-TLE (black line) and CATS track prediction (full/dashed red lines after/before $\delta t_{C A T S}$ correction) and HiSCORE events (blue dots). (b) Black line: LIDAR-ground spot distance from HiSCORE vs. time; blue dots: HiSCORE events MaxAmplitude vs. time (right y-axis). Adjustment done by $\delta t_{T L E}$ for max amplitude at min. distance.

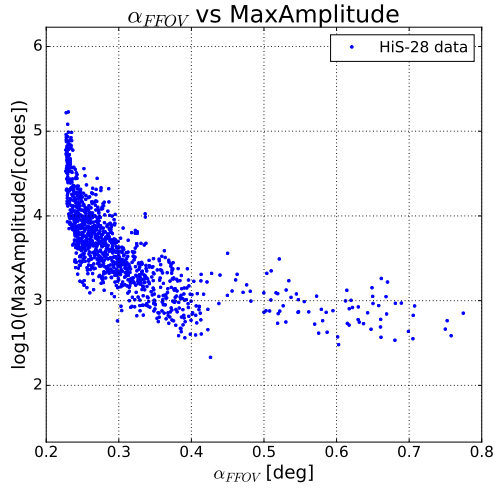

(a)

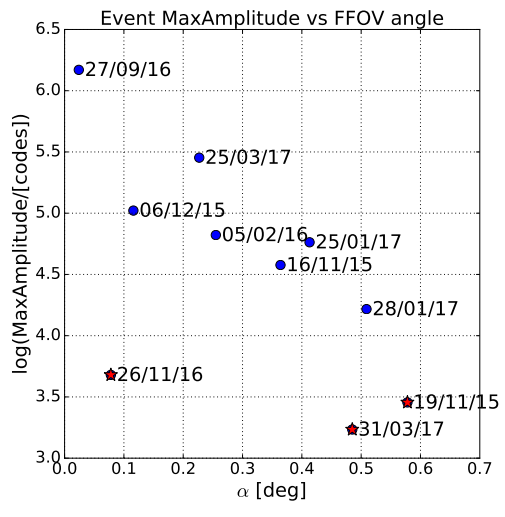

(b)

Figure 7: HiSCORE-CATS combined analysis. (a) Amplitude versus angular distance to the laser beam (viewing angle) $\alpha_{F F O V}$ for ISS-2017/03/25. (b) Max.Amplitude vs. minimal viewing angle $\alpha_{F F O V}$ for all ISS passages (three cloudy passages, see text).

- perform low level technical checks, like: array performance at large illumination, optimization of raw data extraction procedures (pulse extraction, amplitude definition), identification of small systematic effects (masked by stochastics dominated EAS analysis),

- verification of data-analysis procedures, shower reconstruction, etc.,

- a check of station/array amplitude calibration (investigation in progress).

Additionally, also the TAIGA Imaging Atmospheric Cherenkov Telescopes [3] will profit from the ISS-laser beam.

\section{Conclusions}

We report the first ground observation of the CATS-LIDAR onboard the ISS by the TAIGA- 


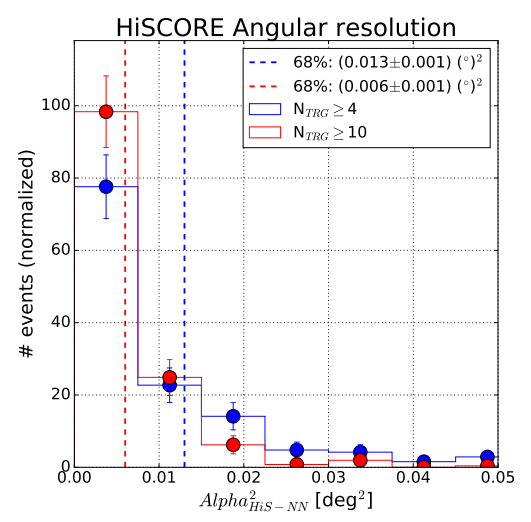

(a)

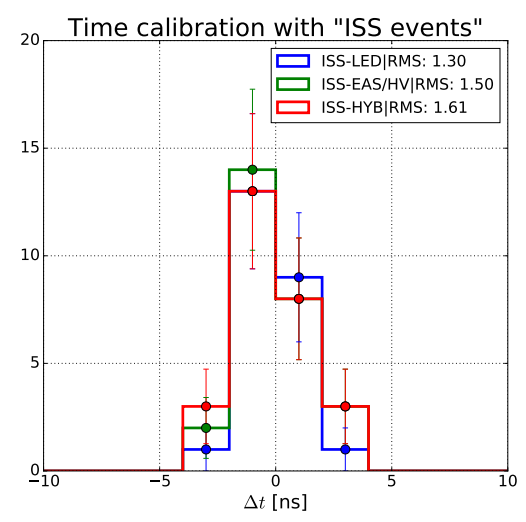

(b)

Figure 8: (a) Angular resolution of HiSCORE for plane wave LIDAR events: space angle $\alpha^{2}$ between neighbouring "ISS events" (station multiplicties $\geq 4$ and $\geq 10$ ). (b) Time-offset calibration using the ISS: comparison between time-offset by ISS and other methods [2].

HiSCORE gamma-ray facility, and by the MASTER-Tunka Robotic telescope. Recorded data are compatible with HiSCORE seeing unscattered laser light events up to $\mathrm{km}$-scale distance to the beam spot, which are following a plane wave front, with nsec jitter.

The ISS-LIDAR turns out to be a unique calibration tool, it establishes a technology to verify absolute astronomical pointing of HiSCORE in combination with MASTER, thus preparing HiSCORE for gamma-ray astronomy. The ISS 'calibration beam' can also be used for a variety of other technical tests and calibrations for HiSCORE. More combined observations will refine the ISS tracking, and allow for precision calibration also with HiSCORE-only detections.

Studying light propagation in the atmosphere by a ground instrument like TAIGA-HiSCORE might complement the ISS-LIDAR technology by detecting the forward scattering component as demonstrated for scattering in dense clouds. We expect, that also other instruments using the atmosphere as active target, like Imaging Air Cherekov Telescopes, will benefit from the ISS light source.

\section{Acknowledgements}

We acknowledge the support of the Russian Federation Ministry of Education and Science (projects: 14.B25.31.0010, 14.593.21.0005, 3.10131.2017/NM, 3.9678.2017/8.9, 3.904.2017/4.6, 3.6787.2017/7.8, 3.6790.2017/7.8), the Russian Foundation for Basic Research (grants 16-0200738, 16-32-00329, 17-02-00905) and the Russian Science Foundation (grant 15-12-20022, sect.2 and 3), from the German Helmholtz Association (grant HRJRG-303), and the DFG (grant TL 513), and from the European Union's Horizon 2020 programme under grant agreement No. 653477. We thank M.McGill and A.Kupchock for very useful discussions and help regarding CATS.

\section{References}

[1] N. Budnev et al., Journal of Physics: Conference Series 718, 052006 (2016) 
[2] M. Tluczykont, et al. (TAIGA-Collaboration), TAIGA-HiSCORE: results from the first two operation seasons, these proceedings

[3] N. Budnev, et al. (TAIGA collaboration), TAIGA - a hybrid detector complex for high energy gamma-ray astro-physics and cosmic ray physics in the Tunka valley, These proceedings

[4] M. Tluczykont, D. Hampf, D. Horns, D. Spitschan, L. Kuzmichev, V. Prosin, C. Spiering, R. Wischnewski, Astroparticle Physics 56, 42 (2014)

[5] N.Budnev et al, (TAIGA-Collabroation), Proc. of INSTR17 Conference Novosibirsk, Russia, 2017

[6] M. Tluczykont, N. Budnev, I. Astapov, et al. (TAIGA-Collaboration), European Physical Journal Web of Conf. 136, 03008 (2017)

[7] L. Sveshnikova et al. (TAIGA collaboration), The search for gamma emission above $50 \mathrm{TeV}$ from Crab Nebula in the TAIGA observatory, These proceedings

[8] A. Porelli, R. Wischnewski, M. Tluczykont, A hybrid time calibration method for EAS ground-based timing arrays, These proceedings

[9] F. Hoots, R. Roehrich, SPACETRACK REPORT NO. 3 Models for Propagation of NORAD Element Sets, (1980)

[10] http://spaceflight.nasa.gov/realdata/sightings/SSapplications/Post/JavaSSOP/SSOP_Help/tle_def.html

[11] http://cats.gsfc.nasa.gov/

[12] V. Lipunov et al., 2010, MASTER Robotic Net, Advances in Astronomy, vol. 2010, pp. 1-7; and http://observ.pereplet.ru/ 